\title{
Clinical Activity and Safety of Anlotinib Combined with PD-I Blockades for Patients with Previously Treated Small Cell Lung Cancer
}

\author{
Yan-Yan Hao ${ }^{1,2, *}$ \\ Yi-Peng Qiao ${ }^{3, *}$ \\ Jian-De Cheng ${ }^{1,2}$
}

'Department of Respiratory and Critical Care Medicine, Shanxi Bethune Hospital, Shanxi Academy of Medical Sciences, Tongji Shanxi Hospital, Third Hospital of Shanxi Medical University, Taiyuan, 030032, Shanxi, People's Republic of China; ${ }^{2}$ Tongji Hospital, Tongji Medical College, Huazhong University of Science and Technology, Wuhan, 430030, Hubei, People's Republic of China; ${ }^{3}$ Department of Internal Medicine, Qing-Xu County People's Hospital, Taiyuan, 030499, Shanxi, People's Republic of China

*These authors contributed equally to this work
Correspondence: Jian-De Cheng Department of Respiratory and Critical Medicine of Shanxi Bethune Hospital, Shanxi Academy of Medical Sciences, Tongji Shanxi Hospital, Third Hospital of Shanxi Medical University, Taiyuan, 030032, Shanxi, People's Republic of China

Tel +86 I3834668789

Email chenjiande_200I@I63.com
Objective: Anlotinib was the standard monotherapy for patients with previously treated small cell lung cancer (SCLC) in recent years. Programmed cell death protein 1 (PD-1) blockade combined with antiangiogenic targeted drugs have proved to play a synergistic action for cancer treatment clinically. Consequently, the present study was to investigate the efficacy and safety of anlotinib combined with PD-1 blockades for patients with previously treated SCLC.

Methods: A total of 36 patients with SCLC who were treated with at least one previous systemic chemotherapy regimen participated in this study retrospectively. All the patients were administered with anlotinib plus PD-1 blockades therapy. Clinical activity was assessed according to the change of target lesion by imaging evidence and all the subjects were followed up regularly. Safety profiles were collected and documented during the treatment. Univariate analysis was carried out using Log rank test and multivariate analysis was adjusted by Cox regression analysis.

Results: All the 36 patients with previously treated SCLC were able to have their efficacy and safety profile evaluated. The best overall response of the combination regimen showed that complete response was observed in one patient, partial response was noted in 9 patients, stable disease was reported in 19 patients, progressive disease was seen in 7 patients. Therefore, the objective response rate (ORR) of the 36 patients was $27.8 \%$ (95\% CI: 14.2 $45.2 \%$ ), disease control rate (DCR) was $80.6 \%$ (95\% CI: 64.0-91.8\%). Regarding the prognostic data, the median PFS and OS of the 36 patients was 4.6 months $(95 \% \mathrm{CI}$ : 3.13-6.07) and 9.3 months (95\% CI: 3.30-15.30), respectively. The most common treatmentrelated adverse reactions were hypertension (52.8\%), fatigue (47.2\%), diarrhea $(38.9 \%)$, hand and foot reaction (38.9\%) and dermal toxicity $(33.3 \%)$. Furthermore, multivariate Cox regression analysis for PFS indicated that ECOG performance status was an independent factor to predict PFS.

Conclusion: Anlotinib combined with PD-1 blockades regimen preliminarily demonstrated encouraging efficacy and tolerable safety for patients with previously treated SCLC. The conclusion should be validated in prospective clinical trials subsequently.

Keywords: small cell lung cancer, anlotinib, PD-1 blockade, efficacy, safety

\section{Introduction}

As an aggressive and devastating malignancy, small cell lung cancer (SCLC) is characterized by rapid growth and early metastasis, and accounts for approximately $15 \%$ of all diagnosed lung cancers. ${ }^{1}$ The estimated prevalence of SCLC was approximately 122,000 new cases and 107,000 new deaths of SCLC in China annually. ${ }^{2}$ Almost 70\% 
of the patients with SCLC presented with extensive-stage SCLC (ES-SCLC), which was of dismal prognosis with the 5 -year survival rate $<3 \%$ and median overall survival (OS) of $<12$ months. ${ }^{3}$ To our knowledge, after the failure for first-line regimen of etoposide plus platinum doublet chemotherapy, topotecan remained the recommended second-line treatment in China with the median duration of response only 3 months. ${ }^{4}$ Therefore, effective regimens with tolerable safety profile as second-line and above therapy for patients with ES-SCLC are needed currently.

It was reported that approximately $80 \%$ of SCLC was associated with positive vascular endothelial growth factor (VEGF) expression. ${ }^{5}$ Therefore, antiangiogenic targeted drugs demonstrated encouraging efficacy for patients with ES-SCLC recently. ${ }^{6}$ Additionally, anlotinib was reported to improve PFS and OS dramatically in a phase II clinical trial (ALTER1202) for patients with SCLC. ${ }^{7}$ Consequently, anlotinib had become the standard regimen as third-line monotherapy for patients with SCLC by NMPA since 2019.

Furthermore, SCLC was correlated with tobacco exposure and progressed rapidly, which frequently involved in central nervous system (CNS) metastasis. ${ }^{8}$ Additionally, SCLC was also associated with high tumor mutation burden and patients with SCLC might benefit from PD-1/PD-L1 blockades treatment. ${ }^{9}$ To our knowledge, although the combination of PD-L1 blockade with first-line platinum-etoposide doublet chemotherapy regimen exhibited superior improvement for clinical outcomes of patients with SCLC according to the two randomized phase III clinical trials (atezolizumab in Impower 133 and durvalumab in CASPIAN), ${ }^{10,11}$ the current clinical evidence data suggested that immunotherapy for SCLC still faced considerable challenges, including limited efficacy of immunotherapy, unknown predictive biomarkers, unknown efficacy in elderly and poor performance status patients and controversial application effect of brain/liver metastases. ${ }^{12}$ Consequently, more effective combination treatment regarding immunotherapy was needed to prolong the survival for patients with SCLC urgently. ${ }^{13}$

Interestingly, the combination of immunotherapy with antiangiogenic targeted drugs exhibited a promising synergistic action in cancer treatment, which indicated that the antiangiogenic drugs reprogrammed the tumor milieu from an immunosuppressive to an immune permissive microenvironment. And the activated immunity by PD-1/PD-L1 blockades also facilitated anti-angiogenesis by downregulating the expression of vascular endothelial growth factor and alleviating hypoxia condition. ${ }^{14}$ Furthermore, recent years had witnessed that atezolizumab plus bevacizumab exhibited promising clinical efficacy in unresectable hepatocellular carcinoma, thus becoming the standard treatment as firstline therapy for unresectable hepatocellular carcinoma. ${ }^{15}$ Nevertheless, the clinical activity and safety of anlotinib combined with PD-1 blockades for patients with previously treated SCLC was still scanty. Therefore, the present study was to investigate the efficacy and safety of anlotinib combined with PD-1 blockades for patients with previously treated SCLC retrospectively.

\section{Patients and Methods Design of This Study and Eligibility Criteria}

Given that anlotinib and PD-1 blockades were licensed in China almost 3 years ago, some patients with SCLC had received anlotinib combined with PD-1 blockades therapy clinically, thus our study was designed as a retrospective study. Patients with ES-SCLC who were treated with at least one systemic chemotherapy regimen in the Department of Respiratory and Critical Care Medicine of the Shanxi Bethune Hospital from August 2018 to May 2021 were recruited in this study consecutively. Inclusion criteria were: (a) histological or cytological diagnosis of SCLC with imaging staging of extensive stage; (b) age $\geq 18$ years old; (c) eastern cooperative oncology group (ECOG) performance status (PS) of 0-2 score; (d) patients progressed after at least one systemic chemotherapy regimen, including those who relapsed $>3$ months after the completion of first-line chemotherapy (platinum-sensitive) and those who relapsed $<3$ months after the completion of first-line chemotherapy or during chemotherapy (platinum-resistant); (e) patients were administered with anlotinib plus PD-1 blockades combination therapy; (f) at least one measurable target lesion to present the drug response according to response evaluation criteria in solid tumors (RECIST 1.1). The exclusion criteria were: (a) previous exposure to PD-1/PD-L1 blockades, or anlotinib was administered previously. However, other antiangiogenic targeted drugs exposure was permitted; (b) clinically active brain metastases, patients with stable brain metastases were permitted; (c) active or uncontrolled autoimmune disease; (d) concomitant with another cancer or serious disease that might compromise the survival of the patients; (e) efficacy assessment data were not available. The study profile of the present study is illustrated in Figure 1. Finally, a total of 36 patients with ES-SCLC was enrolled. This study was approved by the ethics committee of the Shanxi Bethune Hospital. Written informed consent was 
A total of 150 patients extensive-stage SCLC were screened retrospectively from June 2018 to

May 2021

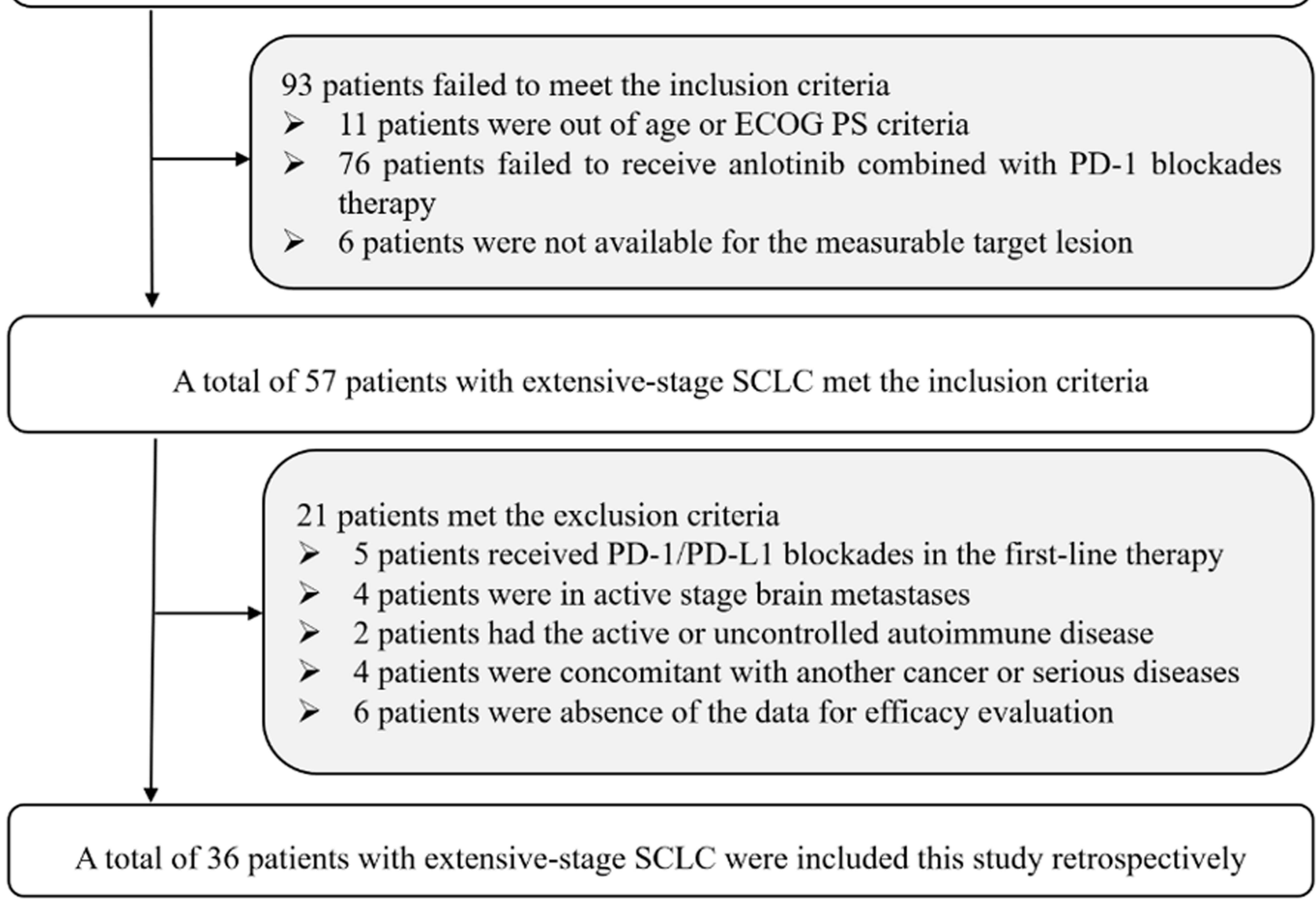

Figure I Flow chart of the retrospective study of anlotinib combined with PD-I blockades in the treatment for patients with previously treated small cell lung cancer.

obtained by each enrolled patient according to the recommendations of the Declaration of Helsinki.

\section{Therapeutic Regimens}

Patients included were treated with anlotinib combined with PD-1 blockades. Anlotinib was administered orally at an initial dosage of $12 \mathrm{mg}$ or $10 \mathrm{mg}$ per day with warm water for two weeks and discontinued for one week, every three weeks as one cycle. PD-1 blockades consisted of camrelizumab (200 mg), sintilimab (200 mg) and pembrolizumab (200 mg), which were intravenously administered on day 1 , every three weeks as one cycle. The treatment continued until disease progression or intolerable adverse reactions. Dosage adjustment of anlotinib to either $10 \mathrm{mg}$ or $8 \mathrm{mg}$ once daily was permitted according to the toxicity during the treatment. Overall response was evaluated according to RECIST version 1.1 criteria based on the judgement of the investigators. ${ }^{16}$
Imaging examination of the target lesions using computed tomography (CT) or magnetic resonance imaging (MRI) was implemented for each patient before and during the combination therapy. The change of target lesions was evaluated using CT or MRI scans every two cycles or depending on the actual situation when it was necessary for the patients. Primary endpoint of this study was progression-free survival (PFS), secondary endpoint was overall survival (OS), objective response rate (ORR), disease control rate (DCR) and safety profile of the combination regimen.

\section{Follow-Up and Assessment of the Safety Profile}

When the patients underwent treatment in the hospital, clinical characteristics, adverse reactions and status of disease progression of each patient were collected through the electronic medical record system. Besides, the subsequent 
follow-up was performed mainly using telephone. Patients were followed up every one month for the treatment after anlotinib combined with PD-1 blockades therapy and death status was mainly inquired. Furthermore, adverse reactions during treatment were documented by Common Terminology Criteria for Adverse Events (CTCAE) 4.03 version to present the toxicity profile that might be drugrelated. ${ }^{17}$

\section{Statistical Analysis}

Data in this study were analyzed using the statistical software SPSS version 25.0 (IBM, USA). ORR was the percentage of complete response (CR) and partial response (PR) in total patients. DCR was the percentage of CR and PR and stable disease (SD) in total patients. PFS was defined as the interval from the onset of anlotinib combined with PD-1 blockades treatment to disease progression or death, whichever occurred first. OS was defined as the interval from the onset of anlotinib combined with PD1 blockades treatment to death of the patients from any cause. ${ }^{18}$ When no prognostic events were observed, survival end points were censored at the date of last follow-up. Kaplan-Meier curves were drawn using Stata version 14.0 to present the PFS and OS data. Survival difference according to baseline characteristic subgroup was calculated using Log rank test. Cox regression analysis was introduced for PFS in multivariable analysis. $P<0.05$ was considered as statistically significant.

\section{Results}

\section{Baseline Characteristics of the 36 Patients with ES-SCLC}

Baseline characteristics of the 36 patients with ES-SCLC was exhibited in Table 1. Median age of the 36 patients was 68 years (range: $21-83$ years), patients with $\geq 68$ and $<68$ years were observed in 20 and 16 cases, respectively. ECOG performance status of $0-1$ and 2 score was observed in 17 and 19 patients, respectively. Male and female was noted in $72.2 \%$ and $27.8 \%$ patients, respectively. Non-smoker and former smoker/smoker was reported in 9 and 27 patients, respectively. Patients with platinum-sensitive and platinumresistant were observed in 17 and 19 cases, respectively. Interestingly, lines of previous treatment with first-line, second-line and subsequent-line were seen in 5, 16 and 15 patients, respectively. Patients with stable brain metastases were observed in 7 cases. A total of 30 patients (83.3\%) had experienced radiotherapy previously. Besides, a total of 6 patients $(16.7 \%)$ were treated with targeted-drugs previously. Interestingly, it should be noted that initial dosage of anlotinib with $12 \mathrm{mg}$ and $10 \mathrm{mg}$ was reported in 25 and 11 patients, respectively. And PD-1 blockades of camrelizumab, sintilimab and pembrolizumab were found in 17,13 and 6 patients, respectively.

\section{Efficacy of the 36 Patients with ES-SCLC Receiving Anlotinib Combined with PD-I Blockades}

All the 36 patients with previously treated SCLC were able to assess the efficacy data. And the best overall response suggested that $\mathrm{CR}$ was observed in one patient, PR was noted in 9 patients, SD was reported in 19 patients, progressive disease was seen in 7 patients. Consequently, ORR of the 36 patients was $27.8 \%$ [95\% confidence interval (CI): $14.2-45.2 \%$ ], DCR was $80.6 \%$ (95\% CI: 64.0-91.8\%). The waterfall plot for the best percentage change in target lesion of the 36 patients with ES-SCLC is presented in Figure 2. Most of the target lesions among the 36 patients with ES-SCLC shrank dramatically. Interestingly, we observed one patient with SCLC achieved CR after the administration of anlotinib combined with sintilimab regimen. This was a female patient with extensive stage SCLC and ECOG performance status of 0 score. She had received etoposide plus carboplatin as first-line therapy and achieved PR after the first-line therapy and progressed after 8 months response. Then she was included in the present study. The CT scan of the target lesion in lung site before and after the combination therapy is illustrated in Figure 3. The target lesion disappeared completely after the therapy of anlotinib combined with sintilimab. Additionally, all non-target lesions disappeared as well. This patient benefited from the combination administration significantly.

\section{Prognosis of the 36 Patients with ES-SCLC Receiving Anlotinib Combined with PD-I Blockades}

Follow-up was implemented in this study appropriately. The last follow-up date of the present study was June 30, 2021. The median follow-up duration for the 36 patients with ES-SCLC from the date of treatment to the date of data cut-off was 8.5 months (follow-up range: 0.6-23.5 months). As illustrated in Figure 4, the median PFS of the 36 patients with ES-SCLC receiving anlotinib combined with PD-1 blockades was 4.6 months (95\% CI: 3.13-6.07). And the 6-month and 12-month 
Table I Baseline Characteristics of the 36 Patients with ES-SCLC

\begin{tabular}{|c|c|c|}
\hline Characteristics & Total Patients $(\mathrm{N}=36)$ & Percentage \\
\hline \multicolumn{3}{|l|}{ Age (years) } \\
\hline Median (range) & $68(21-83)$ & NA \\
\hline$\geq 68$ & 20 & $55 . \%$ \\
\hline$<68$ & 16 & $44.4 \%$ \\
\hline \multicolumn{3}{|l|}{ ECOG PS score } \\
\hline $0-1$ & 17 & $47.2 \%$ \\
\hline 2 & 19 & $52.8 \%$ \\
\hline \multicolumn{3}{|l|}{ Gender } \\
\hline Male & 26 & $72.2 \%$ \\
\hline Female & 10 & $27.8 \%$ \\
\hline \multicolumn{3}{|l|}{ Smoking status } \\
\hline Non-smoker & 9 & $25.0 \%$ \\
\hline Former smoker/smoker & 27 & $75.0 \%$ \\
\hline \multicolumn{3}{|c|}{ Relapse type of first-line regimen } \\
\hline Platinum-sensitive & 17 & $47.2 \%$ \\
\hline Platinum-resistant & 19 & $52.8 \%$ \\
\hline \multicolumn{3}{|c|}{ Lines of previous treatment } \\
\hline First-line & 5 & $13.9 \%$ \\
\hline Second-line & 16 & $44.4 \%$ \\
\hline Subsequent-line & 15 & $41.7 \%$ \\
\hline \multicolumn{3}{|c|}{ Presence of brain metastases } \\
\hline Yas & 7 & $19.4 \%$ \\
\hline No & 29 & $80.6 \%$ \\
\hline \multicolumn{3}{|l|}{ Previous radiotherapy } \\
\hline Yes & 30 & $83.3 \%$ \\
\hline No & 6 & $16.7 \%$ \\
\hline \multicolumn{3}{|c|}{ Previous targeted-drugs therapy } \\
\hline Yes & 6 & $16.7 \%$ \\
\hline No & 30 & $83.3 \%$ \\
\hline \multicolumn{3}{|l|}{ Initial dosage of anlotinib } \\
\hline $12 \mathrm{mg}$ & 25 & $69.4 \%$ \\
\hline $10 \mathrm{mg}$ & 11 & $30.6 \%$ \\
\hline \multicolumn{3}{|l|}{ PD-I blockades } \\
\hline Camrelizumab & 17 & $47.2 \%$ \\
\hline Sintilimab & 13 & $36.1 \%$ \\
\hline Pembrolizumab & 6 & $16.7 \%$ \\
\hline
\end{tabular}

Abbreviations: ES-SCLC, extensive-stage small cell lung cancer; ECOG, Eastern Cooperative Oncology Group; PS, performance status; NA, not available.

PFS rate was 38.9\% (95\% CI: $23.3-54.2 \%)$ and $24.3 \%(95 \%$ CI: $11.7-39.3 \%$ ), respectively. Furthermore, given that followup duration of the present study was accurate and enough, OS was analyzed meanwhile. As shown in Figure 5, the median OS of the 36 patients with ES-SCLC receiving anlotinib combined with PD-1 blockades was 9.3 months $(95 \% \mathrm{CI}$ : 3.30-15.30). And the 6-month and 12-month OS rate was 69.4\% (95\% CI: 51.7-81.8\%) and 47.1\% (95\% CI: $30.3-$ $62.2 \%)$, respectively.

Furthermore, the median PFS and 95\% CI according to baseline characteristic subgroups in univariate analysis were performed. As shown in Table 2, ECOG performance status score and relapse type of first-line regimen were significantly correlated with PFS in univariate analysis, which indicated that the median PFS of patients with ECOG performance status of 0-1 score was dramatically longer than that of patients with a 2 score (median PFS: 5.6 vs 3.8 months, $P=0.021$ ), and the median PFS of patients with platinum-resistant was shorter than that of patients with platinum-sensitive (median PFS: 3.8 vs 5.2 months, $P=0.041$ ). Additionally, it should be noticed that patients with $12 \mathrm{mg}$ initial dosage of anlotinib had a trend 


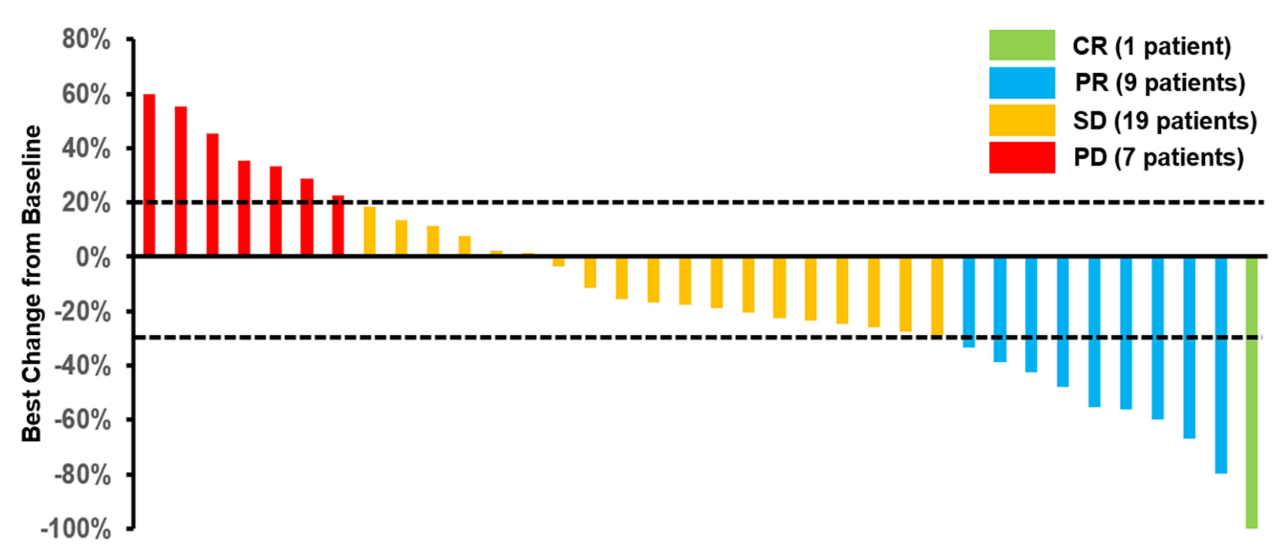

Figure 2 Waterfall plot for the best percentage change in target lesion of the 36 patients with previously treated small cell lung cancer receiving anlotinib combined with PD-I blockades therapy.

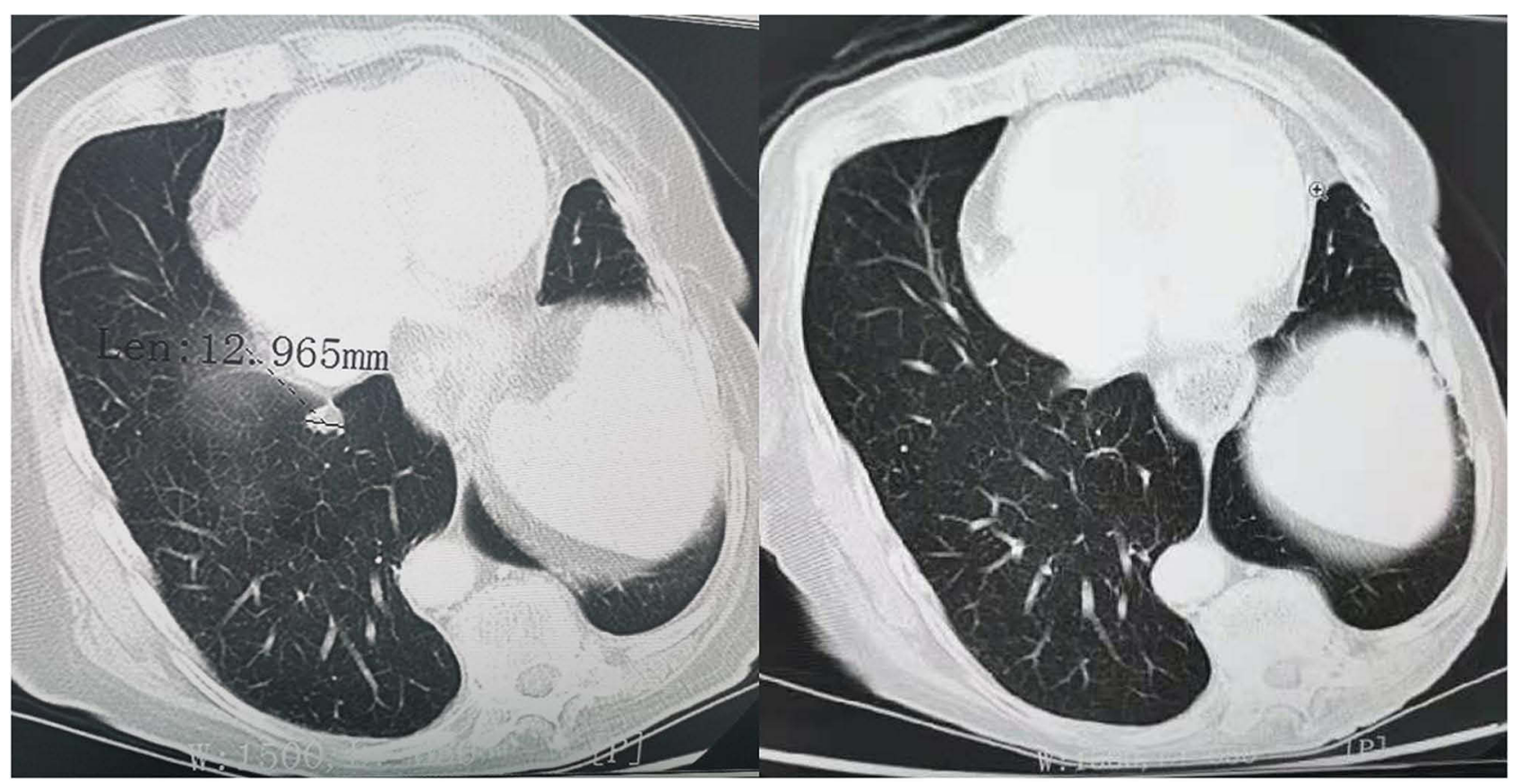

Figure 3 CT scan results of the changes for target lesions in one patient with small cell lung cancer before and after anlotinib combined with PD-I blockade therapy.

for superior PFS compared with those with $10 \mathrm{mg}$ dosage of anlotinib even the difference was not statistically significant (median PFS: 5.2 vs 4.3 months, $P=0.231$ ).

Furthermore, multivariate Cox regression analysis was introduced for PFS adjustment including the baseline characteristics that were significant in univariate analysis. And the multivariate analysis results were illustrated in Table 3. The results demonstrated that ECOG PS score was an independent factor for PFS [hazard ratio $(\mathrm{HR})=0.68$, $P=0.029]$. Nevertheless, as shown in Table 3, after adjustment in the Cox regression analysis, relapse type of first-line regimen failed to influence the PFS independently $(\mathrm{HR}=0.75, P=0.063)$.

\section{Safety Profile of the 36 Patients with ES-SCLC Receiving Anlotinib Combined with PD-I Blockades}

Maximum toxicity of the 36 patients with ES-SCLC experienced during the combination therapy was collected and analyzed in this study. Adverse events regardless of attribution were observed in all the 36 patients (100\%). Nevertheless, the treatment related adverse reactions (TRARs) were noted in 32 patients $(88.9 \%)$. And the grade $\geq 3$ TRARs were seen in 14 patients (38.9\%).

Specifically, as shown in Table 4, the common TRARs were hypertension $(52.8 \%)$, fatigue $(47.2 \%)$, diarrhea 


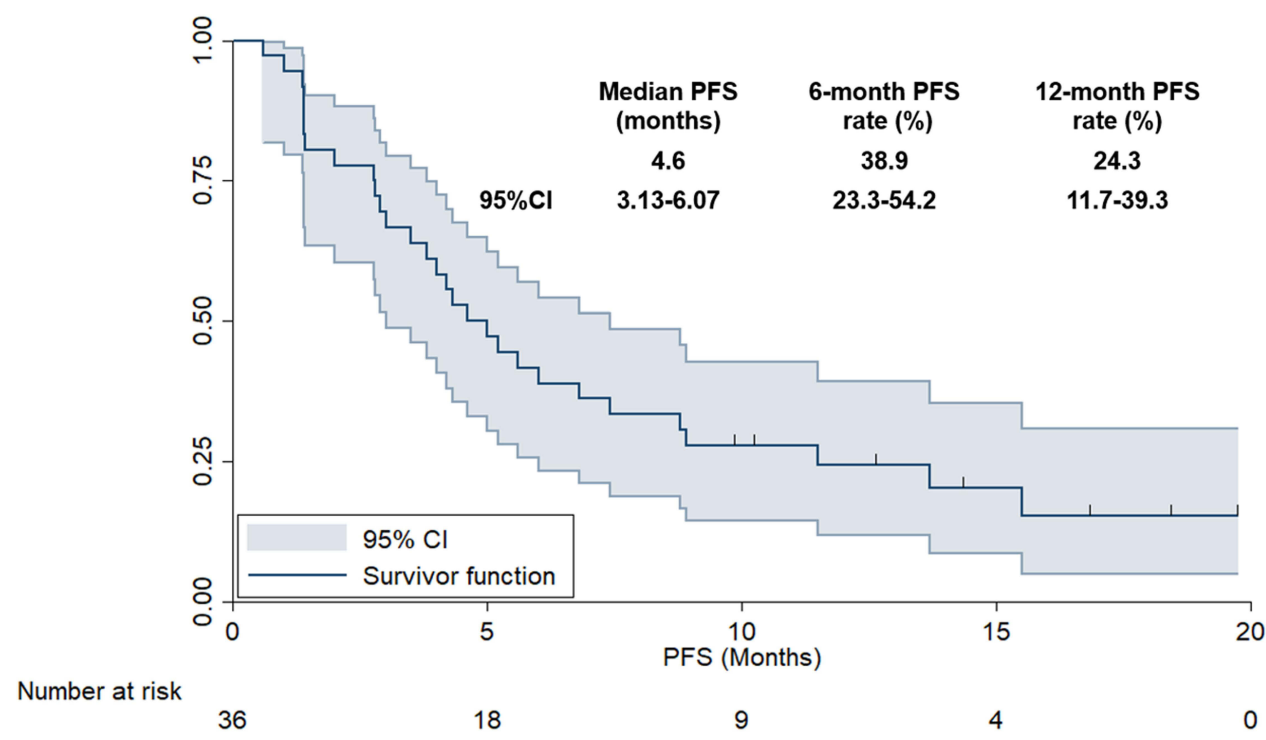

Figure 4 Progression-free survival of the 36 patients with previously treated small cell lung cancer receiving anlotinib combined with PD-I blockades therapy.

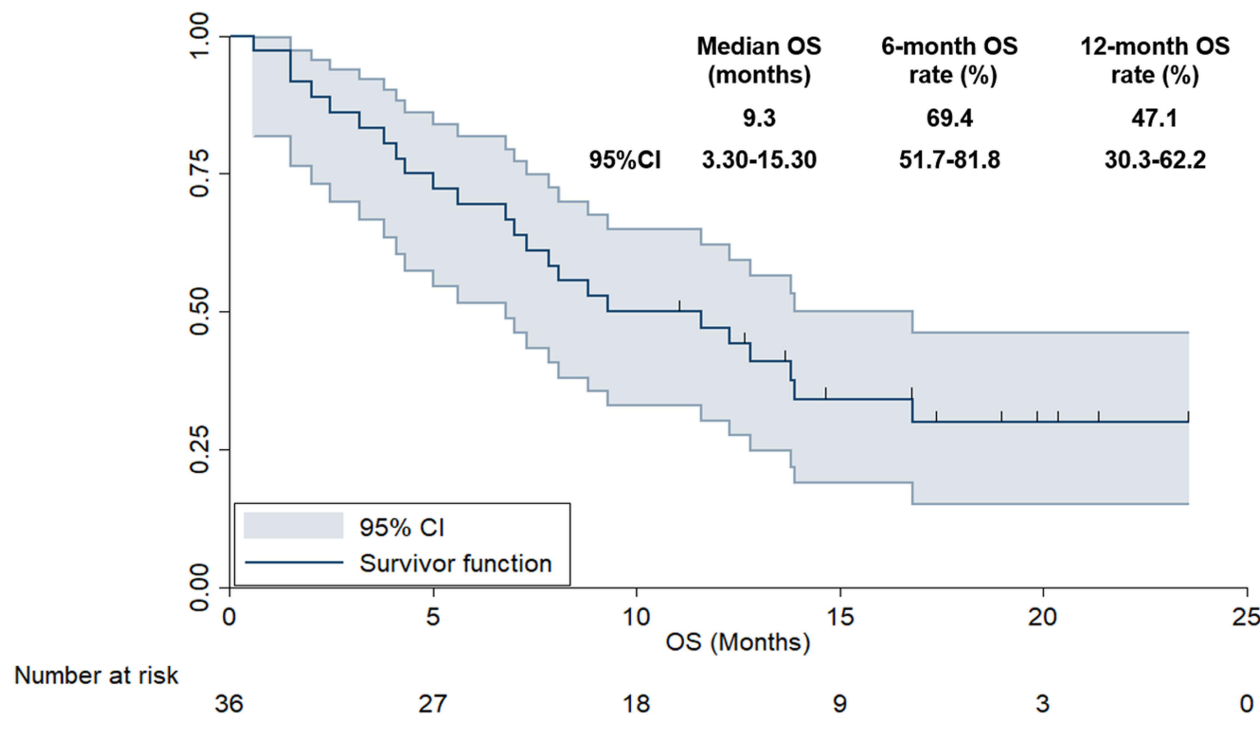

Figure 5 Overall survival of the 36 patients with previously treated small cell lung cancer receiving anlotinib combined with PD-I blockades therapy.

(38.9\%), hand and foot reaction $(38.9 \%)$, dermal toxicity (33.3\%), anemia (25.0\%), ASL/ALT elevation (19.4\%), proteinuria (16.7\%), RCCEP (11.1\%), hemoptysis $(8.3 \%)$ and pneumonia ( $8.3 \%)$. Furthermore, the grade $\geq 3$ adverse reactions were observed in hypertension $(13.9 \%)$, HFS $(11.1 \%)$, diarrhea $(8.3 \%)$, fatigue $(5.6 \%)$, dermal toxicity (5.6\%), AST/ALT elevation (2.8\%) and proteinuria $(2.8 \%)$. Overall TRARs were tolerable and controllable.

\section{Discussion}

To our knowledge, present study highlighted the realworld evidence regarding the clinical activity and safety of anlotinib plus PD-1 blockades for patients with previously treated SCLC. Anlotinib combined with PD-1 blockades could be an effective and safe regimen for patients with previously treated ES-SCLC potentially.

For the past 30 years, etoposide and platinum chemotherapy regimens were widely established as the standard regimen as first-line therapy for patients with SCLC. Although a relatively high response rate of this regimen was observed, SCLC would always relapse inevitably. ${ }^{19}$ The past three years had witnessed that atezolizumab and durvalumab combined with platinum doublet chemotherapy in the firstline setting for patients with ES-SCLC exhibited promising 
Table 2 Univariate Analysis for PFS of the 36 Patients with ES-SCLC According to Baseline Characteristics

\begin{tabular}{|c|c|c|c|c|}
\hline Characteristics & No. of Patients & $\begin{array}{c}\text { Median PFS } \\
\text { (Months) }\end{array}$ & $95 \% \mathrm{Cl}$ & $\boldsymbol{P}$ \\
\hline \multicolumn{5}{|l|}{ Age (Years) } \\
\hline$<68$ & 16 & 4.6 & $3.22-5.98$ & 0.632 \\
\hline$\geq 68$ & 20 & 4.3 & $3.01-5.59$ & \\
\hline \multicolumn{5}{|l|}{ ECOG PS score } \\
\hline $0-1$ & 17 & 5.6 & $4.31-6.89$ & 0.021 \\
\hline 2 & 19 & 3.8 & $2.68-4.92$ & \\
\hline \multicolumn{5}{|l|}{ Gender } \\
\hline Male & 26 & 4.2 & $3.05-5.35$ & 0.438 \\
\hline Female & 10 & 5.0 & $3.93-6.07$ & \\
\hline \multicolumn{5}{|l|}{ Smoking status } \\
\hline Non-smoker & 9 & 5.0 & $3.78-6.22$ & 0.515 \\
\hline Former smoker/smoker & 27 & 4.0 & $3.02-4.98$ & \\
\hline \multicolumn{5}{|c|}{ Relapse type of first-line regimen } \\
\hline Platinum-sensitive & 17 & 5.2 & $3.91-6.49$ & 0.041 \\
\hline Platinum-resistant & 19 & 3.8 & $2.73-4.87$ & \\
\hline \multicolumn{5}{|c|}{ Lines of previous treatment } \\
\hline First-line & 5 & 5.0 & $3.5 \mathrm{I}-6.49$ & 0.417 \\
\hline Second-line & 16 & 4.31 & $3.27-5.35$ & \\
\hline Subsequent-line & 15 & 4.6 & $3.55-5.65$ & \\
\hline \multicolumn{5}{|c|}{ Presence of brain metastases } \\
\hline Yas & 7 & 4.2 & $3.02-5.38$ & 0.336 \\
\hline No & 29 & 5.0 & $3.88-6.12$ & \\
\hline \multicolumn{5}{|l|}{ Previous radiotherapy } \\
\hline Yes & 30 & 4.6 & $3.42-5.78$ & 0.533 \\
\hline No & 6 & 5.0 & $4.06-5.94$ & \\
\hline \multicolumn{5}{|c|}{ Previous targeted-drugs therapy } \\
\hline Yes & 6 & 5.0 & $3.89-6.11$ & 0.423 \\
\hline No & 30 & 4.3 & $3.09-5.51$ & \\
\hline \multicolumn{5}{|l|}{ Initial dosage of anlotinib } \\
\hline $12 \mathrm{mg}$ & 25 & 5.2 & $3.97-6.43$ & 0.231 \\
\hline $10 \mathrm{mg}$ & 11 & 4.3 & $3.08-4.72$ & \\
\hline \multicolumn{5}{|l|}{ PD-I blockades } \\
\hline Camrelizumab & 17 & 4.2 & $3.07-5.33$ & 0.418 \\
\hline Sintilimab & 13 & 5.2 & $3.98-6.42$ & \\
\hline Pembrolizumab & 6 & 5.0 & $3.79-6.21$ & \\
\hline
\end{tabular}

Abbreviations: PFS, progression-free survival; ES-SCLC, extensive-stage small cell lung cancer; ECOG, Eastern Cooperative Oncology Group; PS, performance status; Cl, confidence interval.

clinical activity and tolerable adverse reactions according to Impower133 and CASPIAN clinical trials, respectively. ${ }^{10,11}$ However, treatment in subsequent lines for patients with ESSCLC who failed the standard first-line regimen remained limited. $^{20}$ Although numerous drugs with different mechanisms of action were explored in subsequent line therapy for patients with ES-SCLC, the results were unsatisfactory. ${ }^{21}$ As a novel oral multi-target tyrosine kinase inhibitor, anlotinib inhibited tumor angiogenesis through the target of antiangiogenic signal pathway. ${ }^{22}$ Proliferation of cancer cell was blocked by anlotinib through the newly identified signal pathway involved in the tumor progression. $^{23}$ As a result, anlotinib exhibited promising anticancer activity for patients with previously treated SCLC. ${ }^{24}$ Besides, in spite of the fact that pembrolizumab and nivolumab monotherapy demonstrated potential efficacy and tolerable safety for patients with ES-SCLC over the past three years in phase II trials, ${ }^{25,26}$ the indication for SCLC of pembrolizumab and nivolumab had been withdrawn recently owing to the negative results for OS in the phase III clinical trials. Therefore, these results highlighted the necessity of combination therapy for patients with previously treated SCLC, especially the exploration of PD-1 blockades combined with antiangiogenic targeted drugs. ${ }^{18}$ 
Table 3 Multivariate Cox Regression Analysis for PFS According to Baseline Characteristics

\begin{tabular}{|l|c|c|c|}
\hline Characteristics & HR $(95 \%$ CI) & df & $P$ \\
\hline $\begin{array}{l}\text { ECOG PS score } \\
\text { 0-I vs 2 } \\
\begin{array}{l}\text { Relapse type of first-line regimen } \\
\text { Platinum-sensitive vs platinum-resistant }\end{array}\end{array}$ & $0.68(0.37-0.91)$ & I & 0.029 \\
\hline
\end{tabular}

Abbreviations: PFS, Progression-free survival; ECOG, Eastern Cooperative Oncology Group; PS, performance status; HR, hazard ratio; Cl, confidence interval; df, degree of freedom.

Table 4 Safety Profile of the 36 Patients with ES-SCLC Receiving Anlotinib Combined with PD-I Blockades

\begin{tabular}{|l|c|c|}
\hline Adverse Reactions & Total (N, \%) & Grade $\geq \mathbf{3}(\mathbf{N}, \%)$ \\
\hline Hypertension & $19(52.8)$ & $5(13.9)$ \\
Fatigue & $17(47.2)$ & $2(5.6)$ \\
Diarrhea & $14(38.9)$ & $3(8.3)$ \\
Hand and foot reaction & $14(38.9)$ & $4(11.1)$ \\
Dermal toxicity & $12(33.3)$ & $2(5.6)$ \\
Anemia & $9(25.0)$ & $0(0.0)$ \\
AST/ALT elevation & $7(19.4)$ & $1(2.8)$ \\
Proteinuria & $6(16.7)$ & $1(2.8)$ \\
RCCEP & $4(11.1)$ & $0(0.0)$ \\
Hemoptysis & $3(8.3)$ & $0(0.0)$ \\
Pneumonia & $3(8.3)$ & $0(0.0)$ \\
\hline
\end{tabular}

Abbreviations: ES-SCLC, extensive-stage small cell lung cancer; AST, aspartate amino transferase; ALT, alanine aminotransferase; RCCEP, reactive cutaneous capillary endothelial proliferation.

Patients included in the present study was the ES-SCLC who were treated with at least one systemic chemotherapy treatment, which were similar with the baseline characteristics of Keynote 028 clinical trial. ${ }^{27}$ Collectively, ORR of the 36 patients receiving anlotinib combined with PD-1 blockades was $27.8 \%$, DCR was $80.6 \%$ and the median PFS was 4.6 months. And the results were better than that in the trial of topotecan as second-line therapy for patients with SCLC, which yielded an ORR of $21.7 \%$, DCR of $64.5 \%$ and median PFS of 2.8 months. ${ }^{28}$ Another second-line regimen of lurbinectedin achieved an ORR of $35 \%$, DCR of $68 \%$, a median PFS of 3.5 months and a median OS of 9.3 months in patients with previously treated SCLC, ${ }^{29}$ which demonstrated comparable and similar clinical activity compared with the results of our study. Furthermore, to our knowledge, ORR of anlotinib monotherapy for patients with SCLC was $4.9 \%{ }^{7}$ ORR of PD-1 blockades monotherapy was approximately $<20 \%$ regardless of PD-L1 expression. ${ }^{13}$ The overall response of anlotinib plus PD-1 blockades in our study suggested the potentially synergistic action for cancer therapy, which was in accordance with the conclusion observed in patients with hepatocellular carcinoma who were treated with atezolizumab plus bevacizumab combination therapy. ${ }^{15}$ Interestingly, a recent study initiated by Fan et al. performed a multicenter phase II clinical trial to identify the efficacy and safety of camrelizumab (PD-1 blockade) plus apatinib (another antiangiogenic targeted drug) in ES-SCLC. ${ }^{30}$ A total of 59 patients with ES-SCLC who received camrelizumab plus apatinib treatment were included. The ORR of the 59 patients was $34 \%$, which was higher than that in our study. We speculated the retrospective design of our study might contribute to the discrepancy between the two studies. To our knowledge, the adherence of the patients in the retrospective study was inferior to that in clinical trial. Besides, ECOG performance status proportion should be taken into consideration, previous study indicated that poor PS status was associated with worse prognosis. ${ }^{31}$ Patients with ECOG 2 score in our study accounted for $52.8 \%$, which was higher than that in Yun Fan's trial (0.0\%). Also, the multivariate Cox result suggested that ECOG PS status was an independent factor for PFS. The result was consistent with the previous study. ${ }^{32}$ Nevertheless, it should be noted that the OS in present study was slightly longer than that in the phase II clinical trial (median OS: 9.3 vs 8.4 months). One possible reason might be the fact that more patients with platinumresistant (66.0\% vs 52.8\%) were included in the study of Yun Fan et al. The results of PFS according to baseline characteristics in our study suggested that patients with platinumresistant had a trend for worse PFS compared with patients with platinum-sensitive. Furthermore, we speculated that another explanation could be attributed to the license of PD-1/PD-L1 blockades since 2018. To our knowledge, PD1/PD-L1 blockades demonstrated potential benefit for patients with chemotherapy-refractory ES-SCLC in the subsequent line therapy. ${ }^{13}$ As a result, more PD-1/PD-L1 blockades were still available for the patients when they progressed after anlotinib plus PD-1 blockades treatment, thus providing the patients with survival benefits successively. Additionally, a total of 7 patients with stable brain metastases were also included in our study and the relevance 
analysis exhibited that those patients could benefit from anlotinib combined with PD-1 blockades regimen as well, which was consistent with the previous study of anlotinib plus PD-1 blockades in patients with NSCLC. ${ }^{33}$ Furthermore, to our knowledge, the blood-brain barrier limited the delivery of chemotherapy and considerable targeted drugs to the brain. However, T cells could cross the bloodbrain barrier easily, thus highlighting a positive role for immunotherapy in tumor with brain metastases. ${ }^{34}$ Besides, it should be noted that patients with $10 \mathrm{mg}$ anlotinib had a trend for worse PFS compared with those with $12 \mathrm{mg}$ dosage, although the difference was not statistically significant $(P=0.231)$. We speculated this could reflect a possibility that some patients with poor performance status stood a good chance to choose $10 \mathrm{mg}$ anlotinib therapy, thus contributing to the worse PFS to some extent. However, the conclusion should be validated in large-scale prospective trials in the future.

Overall adverse reactions of the combination regimen were acceptable and tolerable, which was consistent with the previous report regarding the combination therapy of anlotinib plus PD-1 blockades in lung cancer. $^{18}$ Interestingly, the incidence of grade $\geq 3$ TRARs was $38.9 \%$, which was lower than that observed in the treatment of camrelizumab plus apatinib (grade $\geq 3$ TRARs was $72.9 \%$ ). It seemed that anlotinib plus PD-1 blockades was more tolerable for the patients with ES-SCLC. Additionally, the most common TRARs of the combination regimen were hypertension, fatigue, diarrhea and hand and foot reaction, which might be attributed to the administration of anlotinib and were consistent with the safety profile of the previous study regarding anlotinib in patients with ES-SCLC. ${ }^{35}$ Other immunotherapy-related adverse reactions such as dermal toxicity, reactive cutaneous capillary endothelial proliferation (RCCEP) and pneumonia were observed with low incidence, which might have resulted from the therapy of PD-1 blockades. RCCEP seemed to be the specific adverse reaction of camrelizumab that was administered in 17 patients in our study. Therefore, the actual incidence of RCCEP for camrelizumab administration could be $23.5 \%$, which was lower than that of the camrelizumab monotherapy in the other cancers (above 60\%). ${ }^{36}$ This discrepancy of the RCCEP incidence could be attributed to the fact that antiangiogenic targeted drugs might play a key role to attenuate the incidence of RCCEP during camrelizumab administration. ${ }^{37}$ Collectively, the safety profile of anlotinib combined with PD-1 blockades was manageable and controllable.
Limitations were observed in the present study, inevitably. Firstly, the sample size was small, only 36 subjects were enrolled. Clinical activity of anlotinib combined with PD-1 blockades still needs to be confirmed in more patients. Secondly, this study was designed as a retrospective study and some bias could not be avoided. Thirdly, we failed to perform the PD-L1 expression examination and the relevance of PD-L1 expression to clinical activity of the combination regimen needs to be explored subsequently. However, overall, we thought the present study was of potential clinical significance to provide realworld evidence regarding anlotinib combined with PD-1 blockades for patients with previously treated ES-SCLC.

\section{Disclosure}

The authors declare that there are no conflicts of interest.

\section{References}

1. Calles A, Aguado G, Sandoval C, Álvarez R. The role of immunotherapy in small cell lung cancer. Clin Transl Oncol. 2019;21 (8):961-976. doi:10.1007/s12094-018-02011-9

2. Guo H, Wei J, Li X, et al. Do socioeconomic factors modify the effects of PM1 and SO2 on lung cancer incidence in China? Sci Total Environ. 2021;756:143998. doi:10.1016/j.scitotenv.2020.143998

3. Wang Y, Zou S, Zhao Z, et al. New insights into small-cell lung cancer development and therapy. Cell Biol Int. 2020;44 (8):1564-1576. doi:10.1002/cbin.11359

4. Hart LL, Ferrarotto R, Andric ZG, et al. Myelopreservation with trilaciclib in patients receiving topotecan for small cell lung cancer: results from a randomized, double-blind, placebo-controlled phase II study. Adv Ther. 2021;38(1):350-365. doi:10.1007/s12325-02001538-0

5. Chen P, Zhu J, Liu DY, et al. Over-expression of survivin and VEGF in small-cell lung cancer may predict the poorer prognosis. Med Oncol. 2014;31(1):775. doi:10.1007/s12032-013-0775-5

6. Montanino A, Manzo A, Carillio G, et al. Angiogenesis inhibitors in small cell lung cancer. Front Oncol. 2021;11:655316. doi:10.3389/ fonc. 2021.655316

7. Cheng Y, Wang Q, Li K, et al. Anlotinib vs placebo as third- or further-line treatment for patients with small cell lung cancer: a randomised, double-blind, placebo-controlled Phase 2 study. $\mathrm{Br}$ $J$ Cancer. 2021;125(3):366-371. doi:10.1038/s41416-021-01356-3

8. Robin TP, Rusthoven CG. Radiosurgery for small-cell lung cancer brain metastases: a review. $J$ Thorac Dis. 2020;12(10):6234-6239. doi: $10.21037 /$ jtd.2020.03.90

9. Ogino A, Choi J, Lin M, et al. Genomic and pathological heterogeneity in clinically diagnosed small cell lung cancer in never/light smokers identifies therapeutically targetable alterations. Mol Oncol. 2021;15(1):27-42. doi:10.1002/1878-0261.12673

10. Horn L, Mansfield AS, Szczesna A, et al. First-line atezolizumab plus Chemotherapy in extensive-stage small-cell lung cancer. $N$ Engl J Med. 2018;379(23):2220-2229. doi:10.1056/NEJMoa1809064

11. Paz-Ares L, Dvorkin M, Chen Y, et al. Durvalumab plus platinum-etoposide versus platinum-etoposide in first-line treatment of extensive-stage small-cell lung cancer (CASPIAN): a randomised, controlled, open-label, Phase 3 trial. Lancet. 2019;394 (10212):1929-1939. doi:10.1016/s0140-6736(19)32222-6 
12. Lim JU, Kang HS. A narrative review of current and potential prognostic biomarkers for immunotherapy in small-cell lung cancer. Ann Transl Med. 2021;9(9):809. doi:10.21037/atm-21-68

13. Huang W, Chen JJ, Xing R, Zeng YC. Combination therapy: future directions of immunotherapy in small cell lung cancer. Transl Oncol. 2021;14(1):100889. doi:10.1016/j.tranon.2020.100889

14. Song Y, Fu Y, Xie Q, et al. Anti-angiogenic agents in combination with immune checkpoint inhibitors: a promising strategy for cancer treatment. Front Immunol. 2020;11:1956. doi:10.3389/fimmu.2020.01956

15. Finn RS, Qin S, Ikeda M, et al. Atezolizumab plus bevacizumab in unresectable hepatocellular carcinoma. $N$ Engl J Med. 2020;382 (20):1894-1905. doi:10.1056/NEJMoa1915745

16. Eisenhauer EA, Therasse P, Bogaerts J, et al. New response evaluation criteria in solid tumours: revised RECIST guideline (version 1.1). Eur J Cancer. 2009;45(2):228-247. doi:10.1016/j.ejca.2008.10.026

17. Miller TP, Fisher BT, Getz KD, et al. Unintended consequences of evolution of the common terminology criteria for adverse events. Pediatr Blood Cancer. 2019;66(7):e27747. doi:10.1002/pbc.27747

18. Zhang X, Zeng L, Li Y, et al. Anlotinib combined with PD-1 blockade for the treatment of lung cancer: a real-world retrospective study in China. Cancer Immunol Immunother. 2021;70(9):2517-2528. doi:10.1007/s00262-021-02869-9

19. Stratigos M, Matikas A, Voutsina A, Mavroudis D, Georgoulias V. Targeting angiogenesis in small cell lung cancer. Transl Lung Cancer Res. 2016;5(4):389-400. doi:10.21037/tlcr.2016.08.04

20. de Jong WK, Ten Hacken NH, Groen HJ. Third-line chemotherapy for small cell lung cancer. Lung Cancer. 2006;52(3):339-342. doi:10.1016/j.lungcan.2006.02.005

21. Morgensztern D, Besse B, Greillier L, et al. Efficacy and safety of rovalpituzumab tesirine in third-line and beyond patients with DLL3-expressing, relapsed/refractory small-cell lung cancer: results from the phase II TRINITY study. Clin Cancer Res. 2019;25 (23):6958-6966. doi:10.1158/1078-0432.ccr-19-1133

22. Lin B, Song X, Yang D, et al. Anlotinib inhibits angiogenesis via suppressing the activation of VEGFR2, PDGFRbeta and FGFR1. Gene. 2018;654:77-86. doi:10.1016/j.gene.2018.02.026

23. Sun Y, Niu W, Du F, et al. Safety, pharmacokinetics, and antitumor properties of anlotinib, an oral multi-target tyrosine kinase inhibitor, in patients with advanced refractory solid tumors. J Hematol Oncol. 2016;9(1):105. doi:10.1186/s13045-016-0332-8

24. Ying cheng Y, Wang Q, Li K, et al. OA13.03 Anlotinib as third-line or further-line treatment in relapsed SCLC: a multicentre, randomized, double-blind phase 2 trial. J Thorac Oncol. 2018;13(10): S351-S352. doi:10.1016/j.jtho.2018.08.308

25. Chung HC, Piha-Paul SA, Lopez-Martin J, et al. Pembrolizumab after two or more lines of previous therapy in patients with recurrent or metastatic SCLC: results from the KEYNOTE-028 and KEYNOTE-158 studies. J Thorac Oncol. 2020;15(4):618-627. doi:10.1016/j.jtho.2019.12.109

26. Antonia SJ, López-Martin JA, Bendell J, et al. Nivolumab alone and nivolumab plus ipilimumab in recurrent small-cell lung cancer (CheckMate 032): a multicentre, open-label, Phase 1/2 trial. Lancet Oncol. 2016;17(7):883-895. doi:10.1016/s1470-2045(16)30098-5
27. Ott PA, Elez E, Hiret S, et al. Pembrolizumab in patients with extensive-stage small-cell lung Cancer: results from the phase Ib KEYNOTE-028 study. J Clin Oncol. 2017;35(34):3823-3829. doi:10.1200/jco.2017.72.5069

28. Ardizzoni A, Hansen H, Dombernowsky P, et al. Topotecan, a new active drug in the second-line treatment of small-cell lung cancer: a phase II study in patients with refractory and sensitive disease. The European Organization for research and treatment of Cancer Early Clinical Studies Group and New Drug Development Office, and the Lung Cancer Cooperative Group. J Clin Oncol. 1997;15 (5):2090-2096. doi:10.1200/jco.1997.15.5.2090

29. Trigo J, Subbiah V, Besse B, et al. Lurbinectedin as second-line treatment for patients with small-cell lung cancer: a single-arm, open-label, phase 2 basket trial. Lancet Oncol. 2020;21(5):645-654. doi:10.1016/s1470-2045(20)30068-1

30. Fan Y, Zhao J, Wang Q, et al. Camrelizumab plus apatinib in extensive-stage SCLC (PASSION): a multicenter, two-stage, phase 2 trial. $J$ Thorac Oncol. 2021;16(2):299-309. doi:10.1016/j. jtho.2020.10.002

31. Foster NR, Mandrekar SJ, Schild SE, et al. Prognostic factors differ by tumor stage for small cell lung cancer: a pooled analysis of North Central Cancer Treatment Group trials. Cancer. 2009;115 (12):2721-2731. doi:10.1002/cncr.24314

32. Rades D, Motisi L, Veninga T, et al. Predictors of outcomes and a scoring system for estimating survival in patients treated with radiotherapy for metastatic spinal cord compression from small-cell lung cancer. Clin Lung Cancer. 2019;20(4):322-329. doi:10.1016/j. cllc.2019.04.005

33. Wang S, Hu C, Xie F, Liu Y. Use of programmed death receptor-1 and/or programmed death ligand 1 inhibitors for the treatment of brain metastasis of lung cancer. Onco Targets Ther. 2020;13:667-683. doi:10.2147/ott.s235714

34. Berghoff AS, Ricken G, Wilhelm D, et al. Tumor infiltrating lymphocytes and PD-L1 expression in brain metastases of small cell lung cancer (SCLC). J Neurooncol. 2016;130(1):19-29. doi:10.1007/ s11060-016-2216-8

35. Wu D, Nie J, Hu W, et al. A phase II study of anlotinib in 45 patients with relapsed small cell lung cancer. Int J Cancer. 2020;147 (12):3453-3460. doi:10.1002/ijc.33161

36. Wang F, Qin S, Sun X, et al. Reactive cutaneous capillary endothelial proliferation in advanced hepatocellular carcinoma patients treated with camrelizumab: data derived from a multicenter phase 2 trial. J Hematol Oncol. 2020;13(1):47. doi:10.1186/s13045-020-00886-2

37. Jiang FE, Zhang HJ, Yu CY, Liu AN. Efficacy and safety of regorafenib or fruquintinib plus camrelizumab in patients with microsatellite stable and/or proficient mismatch repair metastatic colorectal cancer: an observational pilot study. Neoplasma. 2021;68 (4):861-866. doi:10.4149/neo_2021_201228N1415
International Journal of General Medicine

\section{Publish your work in this journal}

The International Journal of General Medicine is an international, peer-reviewed open-access journal that focuses on general and internal medicine, pathogenesis, epidemiology, diagnosis, monitoring and treatment protocols. The journal is characterized by the rapid reporting of reviews, original research and clinical studies
Dovepress

across all disease areas. The manuscript management system is completely online and includes a very quick and fair peer-review system, which is all easy to use. Visit http://www.dovepress.com/ testimonials.php to read real quotes from published authors. 